\title{
A IMPORTÂNCIA DAS QUEDAS NO MESMO NÍVEL ENTRE IDOSOS NO ESTADO DE SÃO PAULO
}

Vilma Pinheiro Gawryszewski

Trabalho realizado no Centro de Vigilância Epidemiológica, Secretaria de Estado da Saúde de São Paulo, São Paulo, SP

*Correspondência:

Av. Dr. Arnaldo, 351

Sala 609

Cerqueira Cesar

São Paulo - SP

CEP: 01246000

\section{RESUMO}

ОвлEтivo. Analisar as características das quedas no grupo etário com 60 anos ou mais, com ênfase nas quedas no mesmo nível, residentes no Estado de São Paulo, a partir da análise das diferentes fontes de informação oficiais.

MÉTodos. Foram analisadas as 1.328 mortes registradas no SIM em 2007, 20.726 internações no SIH/SUS em 2008 e os 359 atendimentos realizados em 24 UEs do Estado de São Paulo em 2007. Um teste de regressão logística foi utilizado para testar associações entre variáveis nos atendimentos em emergências.

Resultados. O sexo masculino preponderou nas mortes $(51,2 \%)$ enquanto o sexo feminino preponderou nas internações $(61,1 \%)$ e atendimentos em emergências $(60,4 \%)$. 0 coeficiente de mortalidade foi 31/100.000 habitantes, aumentando com a idade e atingindo o valor de 110,7/100.000 habitantes na faixa de 80 anos e mais. As quedas no mesmo nível foram responsáveis pela maior proporção de mortes definidas (35\%), nas internações (47,5\%) e também nas emergências (66\%), crescendo de importância com o aumento das faixas etárias. A residência foi o local de ocorrência em $65,8 \%$ dos casos atendidos nas emergências. Os traumatismos de cabeça assumem importância nas mortes; as fraturas de fêmur foram as lesões mais frequentes nas internações e emergências. Nas emergências, as mulheres foram 1,55 vezes significativamente mais prováveis de serem atendidas por uma queda do que pelas outras causas externas que os homens. Comparativamente à faixa de 60 a 69 anos, os indivíduos na faixa de 70 a 79 anos foram 2,10 vezes e os indivíduos de 80 anos e mais foram 2,26 vezes significativamente mais prováveis de serem atendidos por uma queda do que pelas outras causas externas. Não houve diferença estatisticamente significante quanto ao sexo ou faixa etária quando se comparou os indivíduos que sofreram quedas no mesmo nível e outros tipos de queda.

Conclusão. Recomenda-se que a prevenção das quedas entre idosos entre na pauta de discussão das políticas públicas sem mais demora.

UnItermos: Idoso fragilizado. Acidentes por quedas. Mortalidade. Emergências. Causas externas.

\section{INTRODUÇÃO}

O crescimento da população de idosos, em números absolutos e relativos, é um fenômeno mundial e está ocorrendo a um nível sem precedentes. ${ }^{1}$ Isto é considerado pela demografia como um sinal de desenvolvimento e para a saúde pública uma conquista, pois almejar vida longa é uma aspiração legítima de todo ser humano. Em relação ao Estado de São Paulo, os dados da Fundação SEADE ${ }^{2}$ mostram que o Estado vem apresentando um estreitamento significativo da base de sua pirâmide etária. Entre 1980 e 2006, a estrutura populacional paulista sofreu alterações significativas com decréscimo de $28,2 \%$ na participação de crianças com 14 anos ou menos e aumento de 56,3\% na proporção de idosos com 60 anos ou mais.

Vários estudos nacionais ${ }^{3,4}$ e internacionais ${ }^{5,6,7}$ apontam as quedas como importante causa de mortalidade, morbidade e incapacitações entre a população idosa. Não conhecemos a real magnitude do problema em nosso meio, mas os Estados Unidos da América estimam que a cada 18 segundos um idoso com 65 anos ou mais é atendido por uma lesão decorrente de queda num serviço de emergência. ${ }^{5}$ Mesmo quando provocam lesões menores, elas podem afetar seriamente a qualidade de vida dos idosos levando ao medo de cair com consequente restrição de atividades, mobilidade, diminuição da atividade física, isolamento social e depressão. ${ }^{5,8,9} \mathrm{Em}$ relação às lesões determinadas pelas quedas, dados de internações hospitalares para a população brasileira mostraram que cerca de $70 \%$ dessas lesões são fraturas, especialmente as do fêmur. ${ }^{3}$

As quedas frequentemente ocorrem como um somatório de fatores de risco intrínsecos e extrínsecos, sendo difícil restringir um evento de queda a um único fator de risco ou a um agente 
causal. Uma revisão da literatura ${ }^{10}$ apontou, entre os fatores intrínsecos, a debilidade muscular, alterações da marcha, deterioração cognitiva e capacidade funcional de realizar as atividades da vida diária e atividades instrumentais da vida diária como fator associado às quedas bem como o uso de determinados medicamentos. Estudos realizados no Brasil mostraram, entre os fatores associados, a idade avançada, ${ }^{4}$ sedentarismo, ${ }^{4}$ autopercepção de saúde como sendo ruim ${ }^{4}$, maior número de medicações referidas para uso contínu ${ }^{4}$, comprometimento da visão ${ }^{11}$, dificuldades na realização de atividades diárias ${ }^{11}$.

Em relação às circunstâncias dessas ocorrências, a literatura ${ }^{8,12}$ aponta que proporção considerável dessas quedas ocorre na própria residência do idoso, significando que se trata de eventos relativamente simples, passíveis de serem reduzidos por meio da adoção de programas e medidas de prevenção. Considerando a abordagem da saúde pública às causas externas, torna-se fundamental obter maior número possível de conhecimentos sobre o problema e unir, sistematicamente, dados sobre sua magnitude, características e consequências. 0 presente estudo tem como objetivo analisar as características das quedas no grupo etário com 60 anos ou mais, com ênfase nas quedas no mesmo nível, residentes no Estado de São Paulo, a partir da análise das diferentes fontes de informação oficiais disponíveis.

\section{MÉTodos}

\section{Definições}

Para as finalidades deste estudo e seguindo os parâmetros utilizados nacional e internacionalmente, sobretudo para estudos epidemiológicos, considera-se como idosa a população de 60 anos ou mais. Trata-se de um grupo internamente heterogêneo, estando sujeito a variações decorrentes do aumento da idade em relação ao grau de independência/dependência física, mental e social, além da capacidade laboral e capacidade de recuperação física aos agravos de saúde. Desse modo, os dados de mortalidade e morbidade hospitalar foram analisados segundo as faixas de 60 a 69 anos, 70 a 79 anos e 80 anos e mais.

De acordo com o Centers for Disease Control and Prevention, ${ }^{5}$ uma queda ocorre quando uma pessoa cai no chão ou a outro nível mais baixo de uma forma não intencional. Esta definição não inclui a severidade da lesão.

\section{Fontes e análise dos dados}

As fontes de dados utilizadas foram as consideradas oficiais operadas pelas Secretarias Municipais de Saúde, Secretarias Estaduais de Saúde e Ministério da Saúde. Cada uma delas aporta diferentes tipos de informações se constituindo em importante ferramenta para o conhecimento da carga de violências e acidentes no país, por isso há necessidade de estudá-las em conjunto. Para as informações de mortalidade foi utilizado o banco de dados do Sistema de Informações sobre Mortalidade (SIM), disponibilizado pela Secretaria de Estado da Saúde de São Paulo, composto pelas informações provenientes das declarações de óbito. Foi analisado o último ano disponível, 2007. Primeiramente foram selecionados todos os óbitos classificados no capítulo XX da Classificação Internacional de Doenças Décima Revisão (CID 10), as chamadas causas externas de morbidade e mortalidade. Posteriormente, dentro deste capítulo, foram selecionados os óbitos codificados entre W00 e W19, que pertencem a categoria quedas. Com o propósito de conhecer as lesões que levaram à morte determinadas pelas quedas no mesmo nível foram levantados os dados referentes à natureza da lesão registradas nas linhas A, B ou C da declaração de óbito. Os dados populacionais para a construção dos coeficientes de mortalidade foram provenientes das estimativas do Censo 2000 , disponibilizadas no site do DATASUS/MS.

Para as informações acerca das internações hospitalares realizadas no SUS, foi utilizado o banco do Sistema de Informações Hospitalares do Sistema Único de Saúde (SIH/SUS), construído com os dados que compõem a Autorização de Internação Hospitalar (AlH). Foi analisado o último ano disponível, 2008. Foram estudados os casos classificados no capítulo XX da CID 10, no diagnóstico secundário e neste grupo foram selecionadas as quedas (W00 a W19). Para o estudo das variáveis acerca da natureza da lesão foram estudados os casos classificados no capítulo XIX da CID 10 - Lesões e envenenamentos.

Os dados relativos aos atendimentos em emergências hospitalares são provenientes de 24 emergências selecionadas no Estado de São Paulo que participaram do Sistema Nacional de Vigilância de Violências e Acidentes (VIVA) no ano de 2007, operado pelo Ministério da Saúde em conjunto a Secretaria Estadual da Saúde e Secretarias Municipais de Saúde de Campinas, Diadema, Santo André, Ribeirão Preto, Sorocaba, Jundiaí, S. José do Rio Preto e Guarulhos. Trata-se de uma amostra intencional que é realizada em Unidades de Urgência e Emergência (UES) credenciadas ao SUS, localizadas nos municípios anteriormente citados. A coleta de dados se deu por meio de entrevista as vítimas de violências e acidentes atendidas nos serviços de urgência e emergência durante os 30 dias consecutivos do mês de setembro, em turnos alternados. Mais detalhes sobre a organização e operacionalização desse sistema podem ser encontrados em publicações específicas. ${ }^{13,14}$ Este sistema tem projeto aprovado pela Comissão Nacional de Ética em Pesquisa (CONEP) e por se tratar de ação de vigilância epidemiológica de âmbito nacional, a assinatura do termo de consentimento livre e esclarecido foi substituída por consentimento verbal, obtido pelo paciente ou por seu responsável. No banco de dados composto pelo conjunto das causas externas atendidas naqueles serviços, foram selecionados os casos classificados como quedas.

$\mathrm{Na}$ análise dos dados das UEs, foram utilizados dois testes de regressão logística. O primeiro para verificar se havia associações significativas quanto ao sexo e faixa etária entre os atendimentos decorrentes de quedas e aqueles decorrentes das outras causas externas. Desse modo, a variável dependente foi a queda, compilada em duas categorias ( $\operatorname{sim}=1$ e não $=0$ ). E o segundo para determinar se havia associações significativas quanto ao sexo e faixa etária, entre os atendimentos decorrentes de quedas no mesmo nível e os decorrentes dos outros tipos de quedas. A variável dependente foi a queda no mesmo nível, compilada em duas categorias ( $\operatorname{sim}=1$ e não $=0$ ). As variáveis independentes foram: sexo e faixa etária. Foram calculadas as Odds Ratio (OR) com os respectivos intervalos de confiança (IC) com 95\% de confiança. Os testes foram conduzidos com nível de significância de $5 \%$. As análises foram realizadas no SPSS versão 13.0 for Windows. 


\section{Resultados}

No ano de 2007, ocorreram 4.169 mortes decorrentes do conjunto das causas externas entre idosos residentes no Estado de São Paulo. Nesse grupo as quedas ocuparam o primeiro lugar entre as causas de morte, responsáveis por 1.328 casos, o que representou $31,8 \%$ do total, com coeficiente de 31,0/100.000 habitantes. A Tabela 1 apresenta a mortalidade decorrente de quedas segundo sexo, faixa etária e tipo de queda. É possível verificar que o sexo masculino apresentou as maiores proporções e coeficientes para o total de óbitos neste grupo e para as faixas de 10 a 79 anos. Somente na faixa de 80 anos e mais é que as mulheres predominam na mortalidade decorrente de quedas. Os coeficientes crescem com a idade, atingindo o valor de $110,7 / 100.000$ habitantes.

Embora quase metade das quedas não tenha sido especificada (48\%) na Declaração de Óbito, uma proporção também considerável (35\%) correspondeu às quedas no mesmo nível, indicando que as circunstâncias da ocorrência devem ser relativamente simples. É também possível verificar que a importância proporcional das quedas no mesmo nível cresce com o aumento da faixa etária. A análise dos dados referentes às lesões determinadas pelas quedas no mesmo nível mostrou que um total de 1220 diagnósticos foram registrados nas linhas A, B ou C da declaração de óbito, uma média de 2,6 registros/caso. Não foram incluídos diagnósticos considerados como mal definidos tais como parada cardíaca. O diagnóstico que apareceu o maior número de vezes foi o traumatismo de cabeça, responsável por $15,1 \%$ do total (184 diagnósticos), seguindo-se as fraturas de quadril e da coxa (134 diagnósticos; $11 \%$ do total), pneumonia não especificada $(89 ; 7,3 \%)$, insuficiência respiratória $(73 ; 6 \%)$, septicemias e embolia pulmonar $(71 ; 5,8 \%)$.

Em relação às internações no SIH/SUS, no ano de 2008 foram registradas 20.726 internações decorrentes de quedas, o que representou $60,7 \%$ do total de internações por causas externas entre idosos. A Tabela 2 mostra essas quedas segundo sexo e tipo, sendo possível observar um padrão um pouco diferente da mortalidade, uma vez que a maior proporção de casos foi verificada no sexo feminino, especialmente para as faixas de 70 a 79 anos e 80 anos e mais. As quedas no mesmo nível ocuparam o primeiro lugar, responsáveis por $47,4 \%$ do total, sendo que as quedas por escorregão, tropeção ou passo em falso foram as principais causas bem definidas de internação, respondendo por $22,8 \%$ do total. Por outro lado, o segundo lugar foi ocupado pelas quedas não especificadas (44,3\%). Similar aos dados de mortalidade, as quedas do mesmo nível tendem a aumentar a sua importância proporcional com a idade. O banco utilizado não permitiu o estudo da natureza da lesão determinada pelas quedas, porém sabe-se que para o conjunto das causas externas as fraturas de fêmur foram preponderantes, responsáveis por $29,5 \%$ do total de internações ( 10.016 casos) e os traumatismos intracranianos por $12 \%$ (4.086 casos). A taxa de mortalidade hospitalar para todas as quedas foi $5,3 \%$ enquanto que para as quedas do mesmo nível foi de 5,6\%. A taxa de mortalidade hospitalar determinada pelos traumatismos intracranianos foi $21,8 \%$ enquanto o valor encontrado para as fraturas de fêmur foi 4,6\%. 0 tempo médio de internação dos pacientes devido às quedas foi 6,2 dias.

\begin{tabular}{|c|c|c|c|c|c|c|c|c|c|c|c|c|}
\hline & \multirow{2}{*}{$\begin{array}{c}60 \text { a } 69 \\
\text { anos } \\
\text { No }\end{array}$} & \multirow[b]{2}{*}{$\%$} & \multicolumn{3}{|c|}{$\begin{array}{c}70 \text { a } 79 \\
\text { anos }\end{array}$} & \multicolumn{3}{|c|}{$\begin{array}{l}80 \text { anos } \\
\text { e mais }\end{array}$} & \multicolumn{3}{|c|}{ Total } & \multirow[b]{2}{*}{ Coef. } \\
\hline & & & Coef. & No & $\%$ & Coef. & No & $\%$ & Coef. & No & $\%$ & \\
\hline \multicolumn{13}{|l|}{ Sexo } \\
\hline Masculino & 220 & 76.9 & 20.4 & 233 & 58.8 & 40,7 & 227 & 35.1 & 102.2 & 680 & 51.2 & 36.4 \\
\hline Feminino & 66 & 23.1 & 5.2 & 163 & 41.2 & 21,0 & 419 & 64.9 & 115.8 & 648 & 48.8 & 26.8 \\
\hline \multicolumn{9}{|l|}{ Categoria CID10 } & 110.7 & 1328 & 100.0 & 31.0 \\
\hline Queda no mesmo nível & 71 & 24.8 & 3,0 & 130 & 32,8 & 9,6 & 263 & 40,7 & 45,0 & 464 & 35,0 & 10,8 \\
\hline $\begin{array}{l}\text {-escorregão, } \\
\text { tropeção, passo } \\
\text { em falso }\end{array}$ & 5 & 1.7 & 0,2 & 8 & 2 & 0.6 & 20 & 3.1 & 3.4 & 33 & 2.5 & 0.8 \\
\hline $\begin{array}{l}\text { - outras quedas no } \\
\text { mesmo nível }\end{array}$ & 66 & 23.1 & 2,8 & 122 & 30.8 & 9.0 & 243 & 37.6 & 41.6 & 431 & 32.5 & 10.0 \\
\hline $\begin{array}{l}\text { Queda de cadeira, leito } \\
\text { ou mobília }\end{array}$ & 6 & 2.1 & 0,3 & 12 & 3.1 & 0.9 & 28 & 4.8 & 4.8 & 49 & 3.7 & 1.1 \\
\hline $\begin{array}{l}\text { Queda em escadas ou } \\
\text { degraus }\end{array}$ & 23 & 8 & 1,0 & 25 & 6.3 & 1.9 & 26 & 4.1 & 4.5 & 74 & 5.6 & 1.7 \\
\hline $\begin{array}{l}\text { Outras quedas de um } \\
\text { nível a outro }\end{array}$ & 53 & 18.5 & 2,2 & 37 & 9.4 & 2.7 & 10 & 1.6 & 1.7 & 100 & 7.6 & 2.3 \\
\hline Queda s/especificação & 133 & 46.5 & 5,6 & 189 & 47.7 & 14.0 & 315 & 48.8 & 54.0 & 637 & 48.0 & 14.8 \\
\hline Total & 286 & 100.0 & 12,1 & 396 & 100.0 & 29.3 & 646 & 100.0 & 110.7 & 1328 & 100.0 & 31.0 \\
\hline
\end{tabular}




\begin{tabular}{|c|c|c|c|c|c|c|c|c|}
\hline \multirow[t]{2}{*}{ Faixa etária (anos) } & \multirow{2}{*}{$\begin{array}{c}60 \text { a } 69 \\
\text { No. }\end{array}$} & \multicolumn{3}{|c|}{70 a 79} & \multirow{2}{*}{$\begin{array}{l}80 \mathrm{e}+ \\
\text { No. }\end{array}$} & \multicolumn{3}{|c|}{ Total } \\
\hline & & $\%$ & No. & $\%$ & & $\%$ & No. & $\%$ \\
\hline \multicolumn{9}{|l|}{ Sexo } \\
\hline Masculino & 3741 & 50.5 & 2527 & 36.8 & 1795 & 27.9 & 8063 & 38.9 \\
\hline Feminino & 3673 & 49.5 & 4349 & 63.2 & 4649 & 72.1 & 12671 & 61.1 \\
\hline Queda no mesmo nível & 3297 & 44.5 & 3264 & 47.5 & 3283 & 51.0 & 9844 & 47.5 \\
\hline $\begin{array}{l}\text { - escorregão, tropeção, passo } \\
\text { em falso }\end{array}$ & 1632 & 22.0 & 1597 & 23.2 & 1494 & 23.2 & 4723 & 22.8 \\
\hline - outras quedas no mesmo nível & 1665 & 22.5 & 1667 & 24.3 & 1789 & 27.8 & 5121 & 24.7 \\
\hline $\begin{array}{l}\text { Queda de leito, cadeira ou } \\
\text { mobília }\end{array}$ & 68 & 0.9 & 87 & 1.3 & 107 & 1.7 & 222 & 1.1 \\
\hline $\begin{array}{l}\text { Outras quedas de um nível a } \\
\text { outro }\end{array}$ & 425 & 5.7 & 238 & 3.5 & 204 & 3.2 & 818 & 3.9 \\
\hline Queda s/especificação & 3321 & 44.8 & 3084 & 44.9 & 2775 & 43.1 & 9180 & 44.3 \\
\hline Total & 7410 & 100.0 & 6874 & 100.0 & 6442 & 100.0 & 20726 & 100.0 \\
\hline
\end{tabular}

Para as fraturas de fêmur este valor foi de 7,6 dias e para os traumatismos intracranianos foi de 6,7 dias.

Os dados provenientes das emergências hospitalares (Tabela 3) mostraram que do total de atendimentos em idosos (593), 60,7\% (359 casos) foram decorrentes de quedas. O sexo masculino respondeu por 142 casos $(39,6 \%)$ e o feminino pelos restantes $217(60,4 \%)$. As quedas no mesmo nível foram responsáveis pela maior proporção de casos $(66 \%)$ e similar ao verificado na mortalidade e morbidade hospitalar sua importância cresce com o aumento das faixas etárias. O local de ocorrência mais frequente foi a residência $(65,8 \%)$, seguindo-se a via pública $(23,2 \%)$. As fraturas foram as lesões responsáveis pela maior proporção de atendimentos, $25 \%$ do total (89 casos) com destaque para as fraturas de membros inferiores (42 casos; $12 \%$ do total) e membros superiores (30 casos; $8,6 \%$ do total). Seguem-se as contusões e depois os cortes/perfurações (estes mais comuns na cabeça e face).

Os resultados do teste de regressão logística realizado mostraram que, comparativamente aos homens, as mulheres foram 1,55 vezes significativamente mais prováveis de serem atendidas por uma queda do que pelas outras causas externas. Comparativamente à faixa de 60 a 69 anos, os indivíduos na faixa de 70 a 79 anos foram 2,10 vezes e os indivíduos de 80 anos e mais foram 2,26 vezes significativamente mais prováveis de serem atendidos por uma queda do que pelas outras causas externas. No entanto, o teste não revelou nenhuma diferença estatisticamente significante quanto ao sexo ou faixa etária quando se comparou os indivíduos que sofreram quedas no mesmo nível e outros tipos de queda.

\section{Discussão}

Entre os pontos relevantes deste estudo, podem ser apontados o fato de apresentar conjuntamente dados de mortalidade, internações e atendimentos em emergências, fornecendo um panorama mais abrangente deste problema de saúde. Além disso, mostram uma perspectiva para o Estado como um todo a partir das fontes oficiais de informação. O presente trabalho forneceu evidências da relação entre ocorrência de quedas e idade avançada (tanto na mortalidade quanto na morbidade hospitalar e atendimentos em emergências), o que é consistente com a literatura ${ }^{4,10}$. Quanto ao sexo, os nossos dados mostram maior associação desses eventos com o sexo feminino, o que também foi observado em outros estudos brasileiros ${ }^{4,15}$, porém a revisão da literatura ${ }^{10}$ mostrou resultados contraditórios. A residência como um importante lugar de ocorrência de lesões também é apontado como importante local de ocorrência de quedas. ${ }^{15}$

As taxas brutas de mortalidade encontradas no presente trabalho são similares às encontradas nos Estados Unidos em 2006. No Estado, as taxas do grupo de 60 anos foram 31/100.000, alcançando o valor de 110,7 entre os indivíduos com 80 anos e mais. Nos Estados Unidos, a taxa de mortalidade decorrente de quedas foi de 34,5/100.000 habitantes para os indivíduos com 60 anos e mais; e para a faixa de 80 anos e mais foi de $107,6 .{ }^{16}$

Cabe ressaltar que é a primeira vez que se estuda a natureza da lesão que levou a morte decorrente de queda. Este estudo forneceu evidências da importância dos traumatismos intracranianos tanto na mortalidade quanto na morbidade hospitalar decorrentes de quedas no mesmo nível, o que aponta que as mesmas podem ter decorrido de perda da consciência (síncopes ou tonturas), escorregão em superfície lisa ou molhada (banheiros e cozinhas), alta 
Tabela 3 - Atendimentos decorrentes de quedas (número e \%) entre indivíduos com 60 anos e mais de idade, segundo faixa etária, sexo e tipo de queda. Emergências hospitalares selecionadas, Estado de São Paulo, 2007

\begin{tabular}{|c|c|c|c|c|c|c|}
\hline & & \multicolumn{5}{|c|}{ Faixa etária (anos) } \\
\hline & & & 60 a 69 & 70 a 79 & $\begin{array}{l}80 \mathrm{e} \\
\text { mais }\end{array}$ & Total \\
\hline \multirow[t]{6}{*}{ Sexo } & Masculino & No & 67 & 54 & 21 & 142 \\
\hline & & $\%$ & 46.9 & 39.1 & 26.9 & 39.6 \\
\hline & Feminino & No & 76 & 84 & 57 & 217 \\
\hline & & $\%$ & 53.1 & 60.9 & 73.1 & 60.4 \\
\hline & Total & No & 143 & 138 & 78 & 359 \\
\hline & & $\%$ & 100 & 100 & 100 & 100 \\
\hline
\end{tabular}

\begin{tabular}{|c|c|c|c|c|c|c|}
\hline $\begin{array}{l}\text { Tipo de } \\
\text { queda }\end{array}$ & Mesmo nível & No & 87 & 90 & 56 & 233 \\
\hline & & $\%$ & 62.1 & 66.2 & 72.7 & 66 \\
\hline & Leito, mobília & No & 7 & 11 & 10 & 28 \\
\hline & & $\%$ & 5 & 8.1 & 13 & 7.9 \\
\hline & Escada/degrau & No & 26 & 21 & 4 & 51 \\
\hline & & $\%$ & 18.6 & 15.4 & 5.2 & 14.4 \\
\hline & $\begin{array}{l}\text { Quedas de um } \\
\text { nível a outro }\end{array}$ & No & 15 & 8 & 5 & 28 \\
\hline & & $\%$ & 9.3 & 4.4 & 5.2 & 6.5 \\
\hline & Outros tipos & No & 1 & 5 & 2 & 8 \\
\hline & & $\%$ & 0.7 & 3.7 & 2.6 & 2.3 \\
\hline & $\begin{array}{l}\text { Quedas não } \\
\text { especificadas }\end{array}$ & No & 4 & 1 & 0 & 5 \\
\hline & & $\%$ & 2.9 & 0.7 & 0.0 & 1.4 \\
\hline & Total & No & 140 & 136 & 77 & 353 \\
\hline & & $\%$ & 100 & 100 & 100 & 100 \\
\hline Local de & Residência & No & 86 & 92 & 55 & 233 \\
\hline $\begin{array}{l}\text { ocor- } \\
\text { rência }\end{array}$ & & $\%$ & 61.4 & 67.6 & 70.5 & 65.8 \\
\hline & Via pública & No & 35 & 29 & 18 & 82 \\
\hline & & $\%$ & 25 & 21.3 & 23.1 & 23.2 \\
\hline & Outros & No & 19 & 15 & 5 & 39 \\
\hline & & $\%$ & 13.6 & 11 & 6.4 & 11 \\
\hline & Total & No & 140 & 136 & 78 & 354 \\
\hline & & $\%$ & 100 & 100 & 100 & 100 \\
\hline Natureza & Sem lesão & No & 5 & 14 & 6 & 25 \\
\hline da lesão & & $\%$ & 3.5 & 10.1 & 7.8 & 7 \\
\hline & Contusão & No & 33 & 31 & 16 & 80 \\
\hline & & $\%$ & 23.4 & 22.5 & 20.8 & 22.5 \\
\hline & Corte/perfuração/ & & & & & \\
\hline & laceração & No & 27 & 26 & 20 & 73 \\
\hline & & $\%$ & 19.1 & 18.8 & 26 & 20.5 \\
\hline & Entorse/luxação & No & 37 & 23 & 7 & 67 \\
\hline & & $\%$ & 26.2 & 16.7 & 9.1 & 18.8 \\
\hline & Fratura & No & 29 & 38 & 22 & 89 \\
\hline & & $\%$ & 20.6 & 27.5 & 28.6 & 25 \\
\hline & Outros & No & 10 & 6 & 6 & 22 \\
\hline & & $\%$ & 7.1 & 4.3 & 7.8 & 6.2 \\
\hline & Total & No & 141 & 138 & 77 & 356 \\
\hline & & $\%$ & 100 & 100 & 100 & 100 \\
\hline
\end{tabular}

fragilidade do idoso, entre outras. Dentro disso, um aspecto importante que deveria ser objeto de mais atenção diz respeito ao uso de medicamentos pelo idoso. Revisão da literatura ${ }^{10}$ destacou que os benzodiazepínicos, neurolépticos, antidepressivos e a polimedicação estão associados ao risco aumentado de quedas. Estudo no Brasil também mostrou prevalência de quedas associada com maior número de medicações referidas para uso contínuo. ${ }^{4}$

Em relação às fraturas de fêmur, também evidenciada nas três fontes de informação, cabe ressaltar que a osteoporose, considerada fator de risco para fraturas, tem alta incidência entre as mulheres e é uma doença diagnosticável, tratável e prevenível, sendo que na maioria dos casos a prevenção não é dispendiosa. Estudo realizado na França, concluiu que esta condição tem um impacto significante na mortalidade geral da população daquele país. ${ }^{6}$

Vários trabalhos mostraram que intervenções efetivas apresentam bons resultados na prevenção das quedas. Revisão da base de dados do Cochrane Bone, Joint and Muscle Trauma Group Specialised Register ${ }^{17}$, que incluiu 111 estudos e 55.303 participantes, mostrou que a realização de atividades físicas em grupo, tais como Tai Chi Chuan, exercícios prescritos individualmente para realização em casa, implantação de programas de prescrição de medicação voltados para médicos da atenção básica, realização de cirurgia de catarata podem reduzir o risco de quedas. Ou seja, alguns dos fatores fisiológicos relacionados ao envelhecimento e que contribuem para a ocorrência desses eventos, tais como o declínio da função visual e funções musculoesqueléticas podem ser compensados com intervenções. Também se recomenda a adição, ao exame de saúde rotineiro para essa faixa, de exames que possam identificar fatores de risco para quedas, tais como acuidades auditiva e visual, osteoporose, dificuldades cognitivas, emocionais e na mobilidade.

Por outro lado, um importante aspecto a ser considerado no estabelecimento de medidas e programas de prevenção diz respeito às mudanças de atitude e comportamento verificados nessa faixa nos últimos tempos (o novo idoso). O que por um lado é muito positivo, pois aponta que o idoso busca mais possibilidades e melhor qualidade de vida atualmente, por outro pode trazer aumento no risco de quedas. Reforça essa percepção um estudo realizado na Austrália que mostrou que muitos indivíduos classificados como idosos não consideravam a si próprios como idosos e por isso não tinham conhecimento sobre o seu risco aumentado para quedas ${ }^{3}$. Outro estudo também realizado naquele país concluiu que embora a maioria das pessoas idosas aceitasse campanhas educativas de prevenção de quedas, não as viam como algo pessoalmente relevante. ${ }^{7}$

As fontes de dados utilizadas estão sujeitas a pelo menos três limitações. A primeira delas é quanto ao SIM, principal fonte de dados de mortalidade utilizada no país, que permite o conhecimento da magnitude do problema, porém sem o detalhamento necessário das circunstâncias do evento. A alta proporção de quedas sem especificação aponta má qualidade do preenchimento da declaração de óbito, porém em se tratando de idosos é sabido que entre aqueles que vivem sozinhos e que porventura venham a morrer sem assistência ou testemunhas pode ser mais difícil o esclarecimento das circunstâncias da morte. Por outro lado, para as finalidades deste trabalho, que é evidenciar a importância das quedas no mesmo nível isto não prejudicou as análises realizadas. A segunda diz respeito ao $\mathrm{SIH}$, que utiliza 
como documento básico a Autorização de Internação Hospitalar $(\mathrm{AlH})$, cuja finalidade é o pagamento da internação, o que pode induzir a erros nos registros das causas de internação. Além disso, este sistema cobre somente às internações realizadas no SUS. Os dados da Agência Nacional de Saúde mostram que no ano de 2007 a cobertura de planos de saúde foi de $37,1 \% .{ }^{18}$ A terceira diz respeito aos dados das emergências, que por ser uma amostra de conveniência não são representativos do Estado.

\section{Conclusão}

Em relação às fontes oficiais de dados, considera-se que apesar das limitações apontadas, elas se constituem em fontes importantes de informação acerca das quedas entre idosos, especialmente quando vistas em conjunto, fornecendo um panorama mais completo para a implantação de ações preventivas. Comparativamente à mortalidade, o banco das internações permitiu conhecer melhor os tipos de queda que levam os idosos à hospitalização e o banco de dados provenientes das emergências possibilitou um maior detalhamento das características desses eventos.

Ante aos achados deste estudo, que evidenciam a importância das quedas no mesmo nível como importante causa de morbidade e mortalidade entre os idosos, recomenda-se que a prevenção das quedas entre idosos entre na pauta de discussão das políticas públicas sem mais demora. Espera-se também que estes resultados estimulem, nos profissionais de saúde que realizam atendimento a este grupo, a incorporação de práticas voltadas para a identificação das condições que se configuram em fatores de risco, na correção do que é passível de tratamento e na orientação aos pacientes e familiares.

\section{Conflito de interesse: não há}

\section{SUMMARY}

THE IMPORTANCE OF FALLS ON THE SAME LEVEL AMONG THE ELDERLY in São Paulo state

OBJECTIVE. To analyze characteristics of fall related injuries, with emphasis on falls on the same level, of those with 60 years or more of age,, resident in the state of Sao Paulo, based on three official information sources.

METHODS. A total of 1,328 deaths registered in the Information Mortality System in 2007, 20,726 hospital admissions registered in the Hospitalization Information System in 2008 and 359 visits to 24 different emergency departments (ED) in 2007 were analyzed. A logistic regression model was used to test associations between some variables.

RESULts. More fatal fall victims were male (51.2\%), while females were predominant among hospital admissions (61.1\%) and ED visits (60.4\%). The mortality rate was 31.0/100,000, reaching $110.7 / 100,000$ among those aged 80 years or more. Falls on the same level were responsible for the largest proportion of definite deaths (35.0 \%), hospital admissions (47.5\%) and also ED visits (66.0\%), increasing with the age groups. Residences were the place of occurrence for $65.8 \%$ of the cases in EDs. Head trauma was important among deaths; femur fractures were the most frequent injuries for hospital admissions and ED visits. Compared to men, women were 1.55 times significantly more likely to be attended for a fall than other external causes. Comparatively In comparison to people aged 60 to 69 years, those aged 70 to 79 years and 80 years old or more were 2.10 and 2.26 times, respectively more likely to be fall victims than victims of other external causes. There was no statistically significant difference among people who suffered falls on the same level and other types of falls, for gender and age groups when one compared individuals

CONCLUSION. It is urgent to implement fall prevention programs forto the elderly. [Rev Assoc Med Bras 2010; 56(2): 162-7]

KEY wORDS: Frail elderly. Mortality. Accidental falls. External causes. Emergency service. Hospital.

\section{REFERÊNCIAS}

1. Lima-Costa MF. Epidemiologia do envelhecimento populacional. In: Rouquayrol MZ, Almeida Filho N. Epidemiologia \& saúde. 6ª ed. Rio de Janeiro: MEDSI; 2003.

2. Fundação SEADE. Índice de envelhecimento. [citado 20 ago 2009]. Disponível

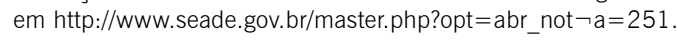

3. Gawryszewski VP, Koizumi MS, Jorge MHP. Mortes e internações por causas externas entre os idosos no Brasil: o desafio de integrar a saúde coletiva e atenção individual. Rev Assoc Med Bras. 2003;50(1):97-103.

4. Siqueira FV, Facchini LA, Piccini RX, Tomasi E, ThuméE, Silveira DS, et al . Prevalência de quedas em idosos e fatores associados. Rev Saúde Pública. 2007;41(5):749-56.

5. Centers for Disease Control and Prevention. Self-reported falls and fall-related injuries among persons aged $\geq 65$ years --- United States, 2006. MMWR. 2008;57(9):225-9.

6. Ziade N, Jougla E, Coste J. Using vital statistics to estimate the population-level impact of osteoporotic fractures on mortality based on death certificates, with an application to France (2000-2004).BMC Public Health. 2009;17;9(1):344.

7. Hughes K, Van Beurden E, Eakin EG, Barnett LM, Patterson E, Backhouse J, et al. Older persons perception of risk of falling: implications for fall-prevention campaigns. Am J Public Health. 2008;98(2):351-7.

8. Fabrício SCC, Rodrigues RA, Costa Junior ML. Causas e conseqüências de quedas de idosos atendidos em hospital público. Rev Saúde Pública. 2004:38(1):9399.

9. Lopes KT, Costa DF, Santos LF, Castro DP, Bastone AC. Prevalência do medo de cair em uma população de idosos da comunidade e sua correlação com mobilidade, equilíbrio dinâmico, risco e histórico de quedas. Rev Bras Fisioter. 2009;13(3):223-9.

10. Gama ZAS, Gómez-Conesa A. Factores de riesgo de caídas en ancianos: revisión sistemática. Rev Saúde Pública. 2008;42(5): 946-956.

11. Perracini MR, Ramos LR. Fatores associados a quedas em uma coorte de idosos residentes na comunidade. Rev Saúde Pública. 2002;36(6):709-16.

12. Douglas AY, Mehan TJ, Collins CL, Smith GA, McKenzie LB. Acute computerrelated injuries treated in U.S. emergency departments, 1994-2006.Am J Prev Med. 2009;37(1):24-8.

13. Gawryszewski VP, Silva MMA, Malta DC, Mascarenhas MDM, Costa VC, Matos SG, et al. A proposta da rede de serviços sentinela como estratégia de vigilância de violências e acidentes. Ciênc Saúde Coletiva. 2007;11(Supl):1269-78.

14. Mascarenhas MDM; Silva MMA; Malta DC; Moura L; MacárioEM;Gawryszewski VP; et al. Perfil epidemiológico dos atendimentos de emergência por violência no Sistema de Serviços Sentinelas de Vigilância de Violências e Acidentes (Viva) - Brasil, 2006. Epidemiologia Serviços Saúde. 2009;18:17-28.

15. Northridge ME, Nevitt MC, Kelsey JL, Link B. Home hazards and falls in the elderly: the role of health and functional status. Am J Public Health. 1995;85(4):509-15.

16. Centers for Disease Control and Prevention. Web-based Injury Statistics Query and Reporting System (WISQARS) [Online]. (2003). National Center for Injury Prevention and Control, Centers for Disease Control and Prevention (producer). [cited 2009 aug 28]. Available from: http://www.cdc.gov/ncipc/wisqars.

17. Gillespie LD, Robertson MC, Gillespie WJ, Lamb SE, Gates S, Cumming RG, et al. Interventions for preventing falls in older people living in the community. Cochrane Database Syst Rev. 2009;(2):CD007146. Review.

18. Agência Nacional de Saúde. Cobertura de planos de saúde. [citado 24 jul 2009]. Disponível em: http:// www.datasus.gov.br.

Artigo recebido: 03/10/09

Aceito para publicação: 17/01/10 